\title{
Some Consequences of a Simple Approach for Constructing a Theory of a Relativistic Fermi Gas
}

\author{
Roberto Lopez-Boada ${ }^{1}$, Luis Grave de Peralta ${ }^{2,3^{*}}$ \\ ${ }^{1}$ Science and Wellness Department, Broward College, Pembroke Pines, FL, USA \\ ${ }^{2}$ Department of Physics and Astronomy, Texas Tech University, Lubbock, TX, USA \\ ${ }^{3}$ Nano Tech Center, Texas Tech University, Lubbock, TX, USA \\ Email: *luis.grave-de-peralta@ttu.edu
}

How to cite this paper: Lopez-Boada, R. and Grave de Peralta, L. (2021) Some Consequences of a Simple Approach for Constructing a Theory of a Relativistic Fermi Gas. Journal of Modern Physics, 12, 19661974.

https://doi.org/10.4236/jmp.2021.1214113

Received: November 25, 2021

Accepted: December 20, 2021

Published: December 23, 2021

Copyright (๑) 2021 by author(s) and Scientific Research Publishing Inc. This work is licensed under the Creative Commons Attribution International License (CC BY 4.0).

http://creativecommons.org/licenses/by/4.0/

\begin{abstract}
It is shown how to build a simple but exact theory of a relativistic Fermi gas at $0{ }^{\circ} \mathrm{K}$, which is based in a recently reported analytic formula for the energies of a relativistic spin- 0 particle in a box. A white dwarf star is then simulated as a sphere filled with a relativistic Fermi gas. The Chandrasekhar mass limit is simply obtained using this model. We then discuss, using the proposed approach to relativistic quantum mechanics, how the interplay between the special theory of relativity, quantum mechanics, and gravity determines the stability of the matter.
\end{abstract}

\section{Keywords}

Quantum Mechanics, Relativistic Fermi Gas, Relativistic Quantum Mechanics

\section{Introduction}

The theory of a non-relativistic Fermi gas is commonly discussed in the context of the theory of metals in solid state physics [1]. The theory of a non-relativistic Fermi gas is built from a well-known analytical expression, which gives the energies of a non-relativistic spin- 0 particle in a three-dimensional infinite well (particle in a box) [2]. The Pauli exclusion principle is used, in the non-relativistic theory of a Fermi gas at $0{ }^{\circ} \mathrm{K}$, for taking care of the fermion nature of the particles forming a Fermi gas [1] [2]. However, this relatively simple theory cannot be directly extended to the relativistic domain [3] [4]. This is because no such analytical expression was known, until recently, for the energies of a relativistic spin-0 particle in a box. In this work, we show, for the first time, how a simple 
but precise theory of a relativistic Fermi gas can be constructed. We use for this a recently reported analytical expression for the energies of a relativistic particle in a box [5] [6] [7] [8]. As it is shown in the Appendix, this analytical expression was obtained by solving a surprising Schrödinger-like but quasi-relativistic wave equation; therefore, using the same mathematical techniques required for obtaining the non-relativistic formula [2] [5] [6] [7] [8] [9].

In what follows, first, in Section 2, some results of such a simple theory of a relativistic Fermi gas, at a $0{ }^{\circ} \mathrm{K}$ temperature, are presented. While the consequences for solid state physics of this theory will be presented elsewhere, here, in Section 3, these results are used, for the first time, for obtaining in an alternative way, simple but precise, the Chandrasekhar mass limit of Fermi gas stars. Then, in Section 4 is presented a discussion about how the interplay between quantum mechanics, Newtonian gravity, and special relativity determines the stability of the matter. Finally, the conclusions of this work are given in Section 5.

\section{The Approach}

The theory of a non-relativistic Fermi gas, formed by $N$ non-interacting fermions with spin-1/2, is based on the knowledge of the analytic expression of the energies corresponding to a 0 -spin particle trapped in an infinite well (particle in a box), which can be easily calculated using the Schrödinger equation [1] [2]. The fermion nature of the particles is included by considering the Pauli exclusion principle, which is purely quantum in nature, and implies that only two fermions can simultaneously be at the same quantum state [1] [2].

Only recently, an analytic expression giving the energies of a spin-0 particle trapped in an infinite well was reported [5] [6] [7] [8]. This result was obtained using the GPPP (Grave de Peralta-Poirier-Poveda) method [5] [6] [7] [8] [9]. A fast review of the GPPP method is described for self-suffice reasons in the Appendix. It is worth noting that, first, there is an excellent agreement between the energies calculated using the analytic formula [5] [6] [7] [8], and the energies numerically calculated using the Dirac equation [10]. Second, it is well understood the relationship between the GPPP method and the Dirac equation [11]. Consequently, we can now construct a simple but exact theory of Fermi gases, which is valid from the non-relativistic to the ultrarelativistic regime. For doing this, we just need to repeat the same steps often follows for constructing the non-relativistic theory of a Fermi gas but using the new energy formula. We should start by substituting the non-relativistic formula for the energies of a quantum particle of mass $m$ in a cubic box of size $L$ [1] [2]:

$$
E_{|\boldsymbol{n}|}=\frac{\pi^{2} \hbar^{2}}{2 m L^{2}}|\boldsymbol{n}|^{2}, \quad \boldsymbol{n}=\left(n_{x}, n_{y}, n_{z}\right), n_{x, y, z}=1,2, \cdots
$$

by the corresponding formula for a relativistic particle [5] [6] [7] [8]:

$$
E_{|n|}=\frac{\pi^{2} \hbar^{2}}{(\gamma+1) m L^{2}}|\boldsymbol{n}|^{2}=(\gamma-1) m c^{2} .
$$


In Equations (1) and (2), $\hbar$ is the reduced Plank constant [1] [2], and $\gamma$ is the especial relativity Lorentz factor [5]-[12]:

$$
\gamma=\sqrt{1+\frac{p_{|n|}^{2}}{m^{2} c^{2}}}=\sqrt{1+|\boldsymbol{n}|^{2} \pi^{2}\left(\frac{\lambda_{C}}{L}\right)^{2}}, \lambda_{C}=\frac{\hbar}{m c} .
$$

In Equation (3), $p$ is the magnitude of the linear momentum of the particle in the box, and $(c)$ is the speed of the light in the vacuum. In the non-relativistic limit, $L \gg \hbar / m c$; therefore $\gamma \approx 1$ and then Equation (2) coincides with Equation (1). In the ultrarelativistic limit, $L \ll \hbar / m c$; therefore $\gamma \gg 1$, thus Equation (3) also gives the correct relation between $E$ and $p$ for an ultrarelativistic particle [3] [4]:

$$
E_{|n|}=p_{|n|} c, \quad p_{|n|}=\frac{|\boldsymbol{n}|}{L} h .
$$

In the thermodynamic limit, $N \gg 1$, each quantum state corresponds to a point in the " $n$-space" with energy given by Equation (2) [1]. In the ground state of the Fermi gas at $0{ }^{\circ} \mathrm{K}$, all the energy levels up to the Fermi energy $\left(E_{F}\right)$ level are occupied, and all the higher levels are empty [1]. The ground state is then represented by a three-dimensional isotropic and uniform Fermi sphere. Therefore, the number of states in the Fermi sphere and its radius are related by the following equation [1]:

$$
N=2 \times \frac{1}{8} \times \frac{4}{3} \pi\left|\boldsymbol{n}_{F}\right|^{3} \Rightarrow\left|\boldsymbol{n}_{F}\right|=\left(\frac{3 N}{\pi}\right)^{1 / 3} .
$$

In Equation (5), the factor of two expresses the two spin states, and the factor of $1 / 8$ expresses the fraction of the sphere that lies in the region where all $n_{x, y, z}$ are positive. Substituting Equation (5) in Equation (2), and replacing $L^{2}$ by $V^{2 / 3}$, we obtain a formula for $E_{F}$ :

$$
E_{F}=\frac{\hbar^{2}\left(3 \pi^{2}\right)^{2 / 3}}{(\gamma+1) m}\left(\frac{N}{V}\right)^{2 / 3}=(\gamma-1) m c^{2}, \gamma=\sqrt{1+\left(3 \pi^{2}\right)^{2 / 3} \lambda_{C}^{2}\left(\frac{N}{V}\right)^{2 / 3}} .
$$

It is straightforward to show that Equation (6) gives the correct values of $E_{F}$ in both the non-relativistic and the ultrarelativistic limits [1] [3] [4]. Moreover, Equation (6) is valid in all this range. The rest of the theory of a Fermi gas is constructed from the analytic formula for $E_{F}[1]$. For instance, the total energy of the Fermi gas when it is in its ground state at $0{ }^{\circ} \mathrm{K}$ is:

$$
E_{T}=\frac{\hbar^{2}}{m}\left(\frac{3 \pi^{2}}{V}\right)^{2 / 3} \int_{0}^{N} \frac{\left(N^{\prime}\right)^{2 / 3}}{\gamma+1} \mathrm{~d} N^{\prime} \approx N \theta E_{F} .
$$

The integral in Equation (7) can be easily calculated in the non-relativistic ( $\theta$ $=3 / 5)$ and the ultrarelativistic $(\theta=3 / 4)$ limits. Consequently, Equation (7) with $\theta$ slowly changing from $3 / 5$ to $3 / 4$ gives the exact value of $E_{T}$ in the relativistic and ultrarelativistic limits [1] [3] [4], and it is a good approximation to $E_{T}$ in between these limits. The degeneracy pressure of the Fermi gas is then calculated as [1] [3] [4]: 


$$
P=-\frac{\partial E_{T}}{\partial V} \propto\left\{\begin{array}{l}
\left(\frac{N}{V}\right)^{\frac{5}{3}}, \text { non-relat } \\
\left(\frac{N}{V}\right)^{\frac{4}{3}}, \text { ultrarelat }
\end{array}\right.
$$

We should emphasize here that the existence of the degenerate pressure in a Fermi gas is a purely quantum effect.

\section{The Chandrasekhar Mass Limit}

Using the Fermi gas as a model, it is possible to calculate the Chandrasekhar limit, i.e., the maximum mass any white dwarf star may have (without significant thermally generated pressure) without collapsing into a black hole or a neutron star. The latter is a star mainly composed of neutrons, where the collapse is also avoided by neutron degeneracy pressure [13] [14].

A simple model for a Fermi gas star, formed by $N \gg 1$ spin-1/2 particles of mass $m$, is a spherical star of radius $r$ formed by a Fermi gas of total mass $M_{S}=$ $N m$ and constant density $\rho=M_{S} / V$, with $V=(4 / 3) \pi r^{3}$. Using this model, the total kinetic energy of the gas (associated to the degeneracy pressure) is $E_{T}$ as given by Equation (7). Consequently, when including the Newtonian gravitational energy [13], we obtain for the total energy of the star $\left(E_{S}\right)$ :

$$
E_{s}(r)=E_{T}(r)-\alpha_{G} \frac{G M_{S}^{2}}{r} \text {. }
$$

In Equation (9), $G$ is the gravitational constant, and the parameter $\alpha_{G}$ is equal to $3 / 5=0.6$ for a constant density sphere, but it takes slightly different values depending on the details of de internal structure of the star $(\rho(r))$. Using Equations (6) and (7), substituting $V$ by (4/3) $\pi r^{3}$, and rearranging Equation (9), we obtain:

$$
E_{s}(r)=\theta a \frac{M_{S}^{5 / 3}}{(\gamma+1) r^{2}}-\alpha_{G} \frac{G M_{S}^{2}}{r}, a=\left(\frac{3 \pi}{4}\right)^{2 / 3} \frac{\hbar^{2}}{m^{8 / 3}}, \gamma=\sqrt{1+\frac{a}{c^{2}} \frac{M_{S}^{2 / 3}}{r^{2}}} .
$$

Or alternatively:

$$
E_{s}(r)=\theta\left(\sqrt{1+\frac{a}{c^{2}} \frac{M_{S}^{2 / 3}}{r^{2}}}-1\right) M_{S} c^{2}-\alpha_{G} \frac{G M_{S}^{2}}{r} .
$$

The radius of the Fermi gas star can be then estimated as the values of $r$ for which $E_{S}(r)$ has a local minimum. When the particles forming the gas move at non-relativistic speeds, it is easier to use Equation (10) with $\gamma=1$. Solving the equation $\mathrm{d} E_{S} / \mathrm{d} r=0$, we obtain:

$$
r=\frac{\theta a}{\alpha_{G} G M_{S}^{1 / 3}}=\left[\left(\frac{3 \pi}{4}\right)^{2 / 3} \frac{\theta}{\alpha_{G}}\right] l_{P}\left(\frac{m_{P}}{m}\right)^{3}\left(\frac{m}{M_{S}}\right)^{1 / 3} .
$$

For obtaining Equation (12), we used the following relations involving the Plank's length $\left(l_{P}\right)$ and mass $\left(m_{P}\right)$ : 


$$
\frac{\hbar^{2}}{G m^{8 / 3}}=\frac{\hbar^{2}}{G m^{3}} m^{1 / 3}, \frac{\hbar^{2}}{G m^{3}}=l_{P}\left(\frac{m_{P}}{m}\right)^{3}, l_{P}=\sqrt{\frac{\hbar G}{c^{3}}}, m_{P}=\sqrt{\frac{\hbar c}{G}} .
$$

Therefore, a non-null radio, which decreases monotonically when $M_{S}$ increases, exists for any star mass. This indicates the existence of an equilibrium between the centrifugal influence of the degeneracy pressure (a purely quantum mechanical effect) and the crushing gravitational force in the massive star. In contrast, when the particles forming the gas move at ultrarelativistic speeds, we can use either one of Equations (10) and (11) with:

$$
\gamma \approx \frac{\sqrt{a}}{c} \frac{M_{S}^{1 / 3}}{r} \gg 1 \text {. }
$$

For obtaining:

$$
E_{S}(r) \approx\left(\theta c \sqrt{a} M_{S}^{4 / 3}-\alpha_{G} G M_{S}^{2}\right) \frac{1}{r}
$$

Consequently, $E_{S} \rightarrow-\infty$ when $r \rightarrow 0$; i.e., the star collapses, when:

$$
M_{S}>M_{C h}=\left(\frac{\theta}{\alpha_{G}}\right)^{3 / 2} \sqrt{\frac{3 \pi}{4}}\left(\frac{m_{P}}{m}\right)^{2} m_{P} .
$$

We obtained an excellent agreement with the Chandrasekhar mass limit $\left(M_{C h}\right.$ $\approx 1.43$ solar mases) [3] [11] [12], when taking $m \approx 1.992$ times the Hydrogen mass $\left(m_{H}\right)$, and $\alpha_{G} \approx 0.4725$. Finally, using Equations (11) and (13), and solving the equation $\mathrm{d} E_{S} / \mathrm{d} r=0$, we obtain:

$$
r=\beta\left[\left(\frac{3 \pi}{4}\right)^{2 / 3} \frac{\theta}{\alpha_{G}}\right] l_{P}\left(\frac{m_{P}}{m}\right)^{3}\left(\frac{m}{M_{S}}\right)^{1 / 3} \sqrt{1-\left(\frac{M_{S}}{M_{C h}}\right)^{4 / 3}} .
$$

The dependence of $r$ (in solar radii) on $M_{S}$ (in solar mases) corresponding to Equation (17) is shown as a continuous red curve in Figure 1. We used here the same values of $m$, and $\alpha_{G}$ reported above. The parameter $\beta=7$ was needed for qualitatively matching previously reported radius-mass relations [3] [14]. This indicates that the inclusion of effects related to the internal structure of the stars are needed for making detailed quantitative calculations [3] [13] [14]. Nevertheless, the overall picture discussed in this Section is correct, and the collapse $(r \rightarrow 0)$ of the ultrarelativistic Fermi gas star in now evident when $M_{S} \rightarrow M_{C h}$. Clearly, Equation (17) coincides with Equation (12) in the non-relativistic limit, i.e., when $M_{S} \ll M_{C h}$. The dependence of $r$ on $M_{S}$ corresponding to Equation (12) is shown as a discontinuous blue curve in Figure 1. Note that the gravitational collapse of a Fermi start can only be predicted when the effects of the especial relativity are included in the model.

\section{Stability of the Matter}

The curves shown in Figure 1 describe the stability of huge cosmological objects formed by numerous quantum particles. However, these curves streakily resemble other curves that were obtained while describing the stability of elemental 


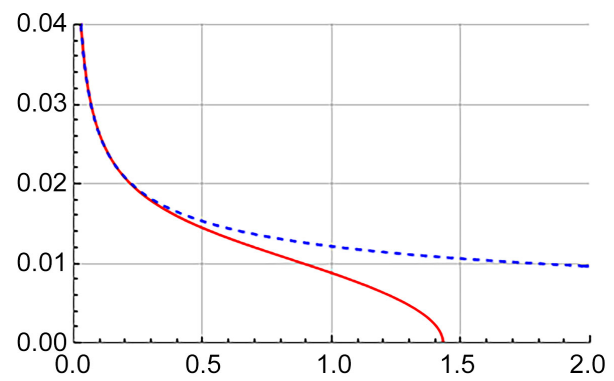

Figure 1. Radius-mass relations in a Fermi gas star model, relativistic (continuous red curve) vs non-relativistic (discontinuous blue curve).

particles and atoms [5]. It has been shown that a good estimate of the size of a Hydrogen-like atom, which is formed by an electron of mass $m_{e}$ bounded to a nucleus of charge $Z e$, where $e$ is the absolute value of the electron charge, can be obtained as the value of $r$ minimizing the energy of the electron in the atom $(E)$, which is given by the following expression [5]:

$$
E(r) \approx \frac{\hbar^{2}}{(\gamma+1) m_{e} r^{2}}-\frac{Z e^{2}}{4 \pi \epsilon_{0} r}, \gamma=\sqrt{1+\left(\frac{\lambda_{C}}{r}\right)^{2}} .
$$

Equation (18) is like Equation (10) but with the relativistic quantum mechanics term, $E_{T}(r)$, substituted by the relativistic quantum mechanics kinetic energy of the electron, and the gravitational energy of the Fermi gas substituted by the Coulombic energy of the electron in the atom [2] [5] [15].

It is not then surprising that the dependence of the size of the atom $(r)$ on $Z$, as shown in Figure 2(a), resembles the curves plotted in Figure 1. In this case, a non-relativistic description (continuous blue curve) predicts atoms with any value of $Z$ should all be stable. However, when special relativity in included in the model (red points), and $Z<137$, the electrical force between the nucleus and the electron tends to collapse the atom but pure quantum mechanical effects stabilize it. In contrast, atoms with $Z>137$ are unstable (the electron collapses to the nucleus, $r \rightarrow 0$ ) and thus these atoms should not exist [5]. Indeed, no atom with $Z>$ 118 has ever been observed.

The following hypothesis, explaining why there are not elemental quantum particles with a mass larger than the Plank mass [5] [16], is much more closely related with the previous discussion about the fate of Fermi gas stars. If an elemental particle of mass $m$ were able to interact gravitationally with itself, due the spread of its mass density through its wave function [5] [16] [17], the energy of the free particle could be estimated using the following equation [5] [16]:

$$
E(r) \approx \frac{\hbar^{2}}{(\gamma+1) m r^{2}}-\frac{G m^{2}}{r} .
$$

With $\gamma$ given by Equation (18). The value of $r$ that minimizes Equation (19) was found solving the equation $\mathrm{d} E / \mathrm{d} r=0$ [5] [16]:

$$
r=a_{D} \sqrt{1-\left(\frac{m}{m_{P}}\right)^{4}}, a_{D}=l_{P}\left(\frac{m_{P}}{m}\right)^{3} .
$$



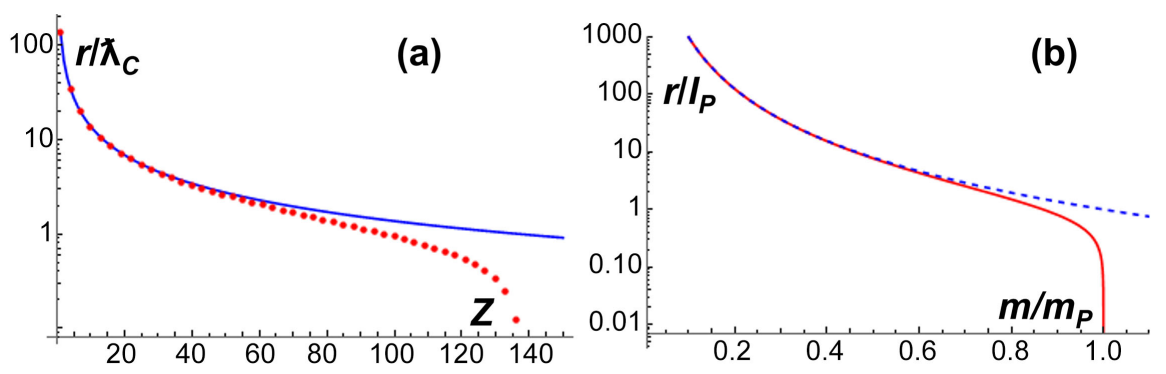

Figure 2. (a) Calculated radii of hydrogen-like atoms in $\lambda_{C}$ (reduced Compton wavelength) units as a function of $Z$ for (continuous blue curve) $\gamma \approx 1$ and (red points) $\gamma \gg 1$. (b) Calculated radii of the radius of a quantum particle, in Plank units, as a function of $m$ in Plank units for (discontinuous blue curve) $\gamma \approx 1$ and (continuous red curve) $\gamma \gg 1$.

In contrast, for the non-relativistic case; i.e., using Equation (19) with $\gamma=1$, it was found that $r=a_{D}$ [5] [16]. As shown in Figure 2(b), a non-relativistic description (discontinuous blue curve) predicts elemental particles with any mass are possible. The dependence of $r$ (in Plank units) on $m$ (in Plank units) corresponding to Equation (20) is plotted in Figure 2(b) as a continuous red curve. When special relativity is included in the model, and $m<m_{B}$ gravity tends to collapse the particle, but pure quantum mechanical effects stabilize it. However, $r \rightarrow 0$ when $m \rightarrow m_{P}$; thus, as it is well known, no elemental particles with mass larger than the Plank mass could exist [5] [16]. Moreover, Figure 2(b) shows that, close to the particle collapse, the size of the particle is $\sim a_{D}$. This factor also appears in Equations (12) and (17). A comparison of Equations (17) and (20), and Figure 1 and Figure 2(b), reveals the transition from stable to instable is more abrupt for a single particle than for a Fermi gas star.

The stability of atoms, the so-called stability of the first kind [18], disappears when the combined effects of special relativity and electrostatic overcome the stability provides by quantum mechanics effects, thus producing the collapse of superheavy atoms. The stability of single quantum particles, a kind of zero-order stability, disappears when the combined effects of special relativity and gravity overcome the stability provided by quantum mechanics effects, thus producing the collapse of elemental particles. While elemental quantum particles with a mass larger than the Plank mass may not exist [5], massive cosmological bodies formed by an extremely large number of quantum particles do exist. The stability of the white dwarfs and neutron stars is an instance of the so-called stability of the second kind [18]. This stability also disappears when the combined effects of special relativity and gravity overcome the quantum effects, associated to the Pauli exclusion principle, that makes the stability possible.

\section{Conclusion}

We have shown that the analytical expression of the energies of a relativistic spin-0 particle trapped in a cubic box, which can be obtained using the GPPP approach (as shown in the Appendix), can be used as the foundation of a simple but exact theory of a Fermi gas, which is valid from the non-relativistic to the ul- 
trarelativistic regimes. For instance, we were able to obtain the Chandrasekar mass limit using this approach and, due to the simplicity of our approach, we were able to illustrate the interplay between gravity, quantum mechanics, and special theory of relativity that determines the stability of matter.

\section{Conflicts of Interest}

The authors declare no conflicts of interest regarding the publication of this paper.

\section{References}

[1] Kittel, C. (2005) Introduction to Solid State Physics. 8th Edition, J. Wiley \& Sons, New York.

[2] Griffiths, D.J. (2018) Introduction to Quantum Mechanics. 3rd Edition, Prentice Hall, New York. https://doi.org/10.1017/9781316995433

[3] Greiner, W., Neise, L. and Stöcker, H. (1997) Thermodynamic and Statistical Mechanics. Springer-Verlag, New York.

[4] Landau, L. and Lifshits, E.M. (1970) Statistical Physics. 2nd Edition, Pergamon Press, Oxford.

[5] Grave de Peralta, L., Poveda, L. and Poirier, B. (2021) European Journal of Physics, 42, Article ID: 055404. https://doi.org/10.1088/1361-6404/ac0ecc

[6] Grave de Peralta, L. (2020) The European Journal of Physics, 41, Article ID: 065404. https://doi.org/10.1088/1361-6404/aba7dc

[7] Grave de Peralta, L. (2020) Results in Physics, 18, Article ID: 103318. https://doi.org/10.1016/j.rinp.2020.103318

[8] Grave de Peralta, L. (2020) Journal of Modern Physics, 11, 196-213. https://doi.org/10.4236/jmp.2020.112012

[9] Grave de Peralta, L. (2020) Scientific Reports, 10, Article No. 14925. https://doi.org/10.1038/s41598-020-71505-w

[10] Ruiz-Columbié, A., Farooq, H. and Grave de Peralta, L. (2021) Journal of Modern Physics, 12, 1358-1374. https://doi.org/10.4236/jmp.2021.1210085

[11] Grave de Peralta, L. and Farooq, H. (2021) Journal of Modern Physics, 12, 1145-1159. https://doi.org/10.4236/jmp.2021.128068

[12] Christodeulides, C. (2016) The Special Theory of Relativity. Springer, New York.

[13] Morison, I. (2008) Introduction to Astronomy and Cosmology. Wiley, London.

[14] Kutner, M.L. (2003) Astronomy, a Physical Perspective. 2nd Edition, Cambridge University Press, Cambridge. https://doi.org/10.1017/CBO9780511802195

[15] Walker, J., Resnick, R. and Halladay, D. (2014) Fundamentals of Physics. 10th Edition, J. Wiley \& Sons, New York.

[16] Poveda, L.A., Ruiz-Columbié, A. and Grave de Peralta, L. (2021) Relativistic Corrections to the Diósi-Penrose Model. http://arxiv.org/abs/2103.00994

[17] Diósi, L. (1984) Physics Letters A, 105, 199. https://doi.org/10.1016/0375-9601(84)90397-9

[18] Lieb, H.E. and Seiringer, R. (2010) The Stability of Matter in Quantum Mechanics. Cambridge University Press, New York. https://doi.org/10.1017/CBO9780511819681

[19] Grave de Peralta, L., Poirier, B. and Poveda, L.A. (2021) APS March Meeting U7100234 . 


\section{Appendix}

The Poirier-Grave de Peralta equation [5] [19]:

$$
i \hbar \frac{\partial}{\partial t} \Psi=\left[\frac{\hat{p}^{2}}{(\hat{\gamma}+1) m}+V\right] \Psi, \hat{\gamma}=\sqrt{1+\frac{\hat{p}^{2}}{m^{2} c^{2}}}, \hat{p}=-i \hbar \nabla .
$$

is fully Lorentz-covariant [5]. It can be shown that for a particle in the box, Equation (A1) exactly reduces to solving the following Schrödinger-like equation [5] [19]:

$$
i \hbar \frac{\partial}{\partial t} \Psi=-\frac{\hbar^{2}}{(\gamma+1) m} \nabla^{2} \Psi+V \Psi
$$

In Equation (A2), $\gamma$ is not an operator but the parameter (GPPP approach) [5]:

$$
\gamma=\sqrt{1+\frac{2}{m c^{2}} E_{S c h}}, E_{S c h}=\left\langle\psi_{S c h}\left|\frac{\hat{p}^{2}}{2 m}\right| \psi_{S c h}\right\rangle .
$$

In Equation (A3), $E_{S c h}$ and $\psi_{s c h}$ are the energy and wavefunction, respectively, of the particle in a box calculated by solving the Schrödinger Equation [2]. Clearly, Equation (A2) is the Schrödinger equation when $\gamma=1$. Moreover, Equation (A2) can be solved as the Schrödinger equation is solved [5] [6] [7] [8] [9]. For a particle in the box [5]:

$$
E_{|\boldsymbol{n}|}=\frac{\pi^{2} \hbar^{2}}{(\gamma+1) m L^{2}}|\boldsymbol{n}|^{2}, \gamma=\sqrt{1+\frac{2}{m c^{2}} E_{S c h}} .
$$

Equations (2) and (3) follows directly from the substitution of $E_{S c h}=E_{|n|}$ given by Equation (1) in Equation (A4). It should be noted that there is an excellent correspondence between the energy values calculated using Equations (2) and (3), and the numerically calculated energies using the Dirac's equation [10]. 